\title{
APLICAÇÃO DE FILTROS BAYESIANOS EM UM ESCOAMENTO BIFÁSICO ÓLEO-ÁGUA EM RESERVATÓRIO DE PETRÓLEO
}

\author{
R. X. HOFFMANN¹, W. B. da SILVA², J. C. S. DUTRA ${ }^{3}$, R. A. G. MARQUES ${ }^{4}$ \\ 1,2,3 Universidade Federal do Espírito Santo, Departamento de Engenharia Rural \\ ${ }^{4}$ Universidade Federal de Alfenas \\ E-mail para contato: 1rayani_hoffmann@hotmail.com, ${ }^{2}$ wellingtonuff@yahoo.com.br, \\ 3juliosdutra@yahoo.com.br, ${ }^{4}$ ramarque13@gmail.com
}

\begin{abstract}
RESUMO - Os problemas de estimação de estado, também conhecidos como problemas inversos não estacionários, apresentam inúmeras aplicações práticas, produzindo estimativas sequenciais das variáveis desejadas através de modelos estocásticos e medidas experimentais, e minimizando estatisticamente o erro. Neste trabalho, o modelo estudado foi de Buckley-Leverett. Suas equações são comumente usadas na modelagem de fluxo de água e óleo imiscíveis em meios porosos. Este estudo é aplicado ao filtro SIR (Sampling Importance Resampling Filter) e ASIR (Auxiliary Sampling Importance Re-sampling Filter) para o conceito de estimativa de estado em um problema de escoamento bifásico (óleoágua) devido à dificuldade significativa associada com a recuperação da saturação do óleo.
\end{abstract}

\section{INTRODUÇÃO}

No Brasil, com a descoberta da camada do pré-sal, viu-se a necessidade de buscar novas tecnologias para a exploração dessas novas jazidas de petróleo. Devido às condições de formação, as rochas reservatórios onde se aloja o petróleo possuem propriedades que dificultam a extração do óleo, tais como a porosidade e permeabilidade. Estes reservatórios são muito heterogêneos e anisotrópicos devido a várias camadas sedimentares depositadas de diferentes formas ao longo do tempo, que criou diversos caminhos preferencias ao fluxo de fluidos diferentes. Por isso, o escoamento do fluido em meio poroso depende das características petrofísicas do meio, as quais não se podem medir diretamente. Desta forma, faz-se necessário o uso de simulações, cujos resultados geram dados que permitem estimar as propriedades do reservatório. Obtêm-se então os parâmetros que descrevem o meio, o qual é considerado um problema inverso. Este tipo de problema pode ser resolvido através dos chamados filtros Bayesianos junto com os problemas de estimação de estados. Nessa abordagem, todas as informações disponíveis são utilizadas, com a finalidade de reduzir a incerteza presente no problema. A base do procedimento estatístico é formada ao combinar a informação anterior com a nova informação obtida. O mecanismo formal utilizado para combinar as informações é conhecido como teorema de Bayes (Vianna et al, 2009; Silva et al, 2010). O presente trabalho aplica os filtros Bayesianos junto ao conceito de estimativa de estado para um problema de escoamento bifásico (óleo-água) que apresenta grande dificuldade relacionada à extração de petróleo. Reiterando, a ampla capacidade e eficácia 
desses métodos em estimar e prever o comportamento dos parâmetros, que podem apresentar um alto grau de incertezas, e escolher o cenário mais adequado para o aumento da produção.

\section{PROBLEMA FÍSICO E FORMULAÇÃO MATEMÁTICA}

Para simular esse processo de engenharia são resolvidas equações diferenciais parciais que constituem o chamado modelo imiscível para escoamentos bifásicos em meios porosos. Essencialmente, esse modelo é formado pelas equações de conservação de massa das fases, água e óleo, juntamente com a conhecida lei de Darcy.

Desprezando-se os efeitos da difusão física, oriunda da pressão capilar, e considerandose um modelo não linear para o chamado fluxo fracionário, a equação de conservação de massa da fase água se reduz a uma lei de conservação hiperbólica não linear. Segundo Buckley e Leverett (1942), a Equação 1 foi formulada para estudar o deslocamento da frente de água durante o processo de recuperação secundária de petróleo.

$$
\frac{\partial S}{\partial t}=-\frac{q_{T} \partial f}{\emptyset \partial x}
$$

onde $S$ é a saturação do fluido deslocado, $q_{T}$ é o fluxo total, $\phi$ é a porosidade, $f$ é a função de fluxo fracionado, $t$ é o tempo e $x$ é a variável espacial. Para o fluxo fracionado utiliza-se geralmente o chamado modelo de Stone (1970), dado pela Equação 2.

$$
f(S)=\frac{S^{2}}{S^{2}+\frac{\mu_{w}}{\mu_{o}}(1-S)^{2}}
$$

onde $\mu_{w}$ é a viscosidade da água e $\mu_{o}$ é a viscosidade do óleo.

\section{PROBLEMA DE ESTIMAÇÃO DE ESTADO}

O problema de estimação de estado pode ser visto como uma versão especial do caso mais geral do problema de estimação do erro médio quadrático, no qual a distribuição a priori para a variável de estado desconhecida (parâmetro a ser estimado) ${ }^{x_{k}}$ no tempo $t_{k}$ é dada por um modelo de evolução temporal (Vianna et al, 2009; Silva et al, 2010). A fim de resolver o problema de estimação de estado, considere o modelo de evolução dado pela Equação 3.

$$
x_{k}=f_{k-1}\left(x_{k-1}, v_{k}\right)
$$

onde o subscrito $k=1,2, \ldots$, representa o instante de tempo $t_{k}$, que varia de um tempo inicial até um tempo final no sistema dinâmico. O vetor $x \in R^{n_{x}}$ é chamado de vetor de estado e contém todas as variáveis que serão estimadas. Este vetor avança no tempo de acordo com o modelo de evolução de estado dado pela Equação 3, na qual $f$ é geralmente uma função não linear das variáveis de estado, e o vetor de incertezas, $v \in R^{n_{v}}$, que representa o vetor de ruído 
do estado (Kaipio e Somersalo, 2004; Vianna et al, 2009; Silva et al, 2010). O modelo de observação ou de medidas é dado pela Equação 4.

$$
z_{k}=h_{k}\left(x_{k}, n_{k}\right)
$$

Em que as medidas são representadas por $z \in R^{{ }^{n} z}$ e o vetor $n \in R^{{ }^{n}}$ que representa o ruído das medidas.

O problema de estimação de estado tem por objetivo obter informações sobre o vetor $x_{k}$ baseado nos modelos de evolução e de observação dados pelas Equações 3 e 4, apoiado nas seguintes suposições (Kaipio e Somersalo, 2004; Vianna et al, 2009; Silva et al, 2010):

(i) A sequência $x_{k}$ para $k=1,2, \ldots$, é um processo Markoviano de primeira ordem (a probabilidade de um estado depende apenas do estado imediatamente anterior), como mostra a Equação 5.

$$
\pi\left(x_{k} \mid x_{0}, x_{1}, \ldots, x_{k-1}\right)=\pi\left(x_{k} \mid x_{k-1}\right)
$$

(ii) A sequência $z_{k}^{o b s}$ para $k=1,2, \ldots$ é um processo Markoviano com respeito a história de $x_{k}$, como na Equação 6.

$$
\pi\left(z_{k}^{o b s} \mid x_{0}, x_{1}, \ldots, x_{k-1}\right)=\pi\left(z_{k}^{o b s} \mid x_{k}\right)
$$

(iii) A sequência $x_{k}$ depende das observações passadas através de sua própria história, ou seja, Equação 7.

$$
\pi\left(x_{k} \mid x_{k-1}, z_{1: k-1}^{o b s}\right)=\pi\left(x_{k} \mid x_{k-1}\right)
$$

Neste contexto, o Teorema de Bayes é dado pela Equação 8.

$$
\pi_{\text {posterior }}\left(x_{k}\right)=\pi\left(x_{k} \mid z_{k}^{o b s}\right)=\frac{\pi\left(x_{k}\right) \pi\left(z_{k}^{o b s} \mid x_{k}\right)}{\pi\left(z_{k}^{o b s}\right)}
$$

onde $\pi\left(x_{k}\right)$ corresponde à densidade priori da variável de estado, $\pi\left(z_{k}^{o b s}\right)$ corresponde à densidade de probabilidade marginal das medidas, que desempenha o papel de uma constante de normalização, $\pi\left(z_{k}^{o b s} x_{k}\right)$ corresponde ao risco (ou seja, a probabilidade condicional das medições $z_{k}^{o b s}$ dadas as variáveis de estado ${ }_{k}$ ).

\subsection{Filtro SIR}

Inicialmente, no filtro SIR (Amostragem por Importância e Reamostragem Sequencial), $N$ partículas são amostradas aleatoriamente de uma distribuição inicial $\pi\left(x_{0}\right)$, que são amostras da distribuição no instante de tempo $t=0$. Para avançar as partículas no tempo $t_{k-1}$ para o tempo $t_{k}, k=1,2,3, \ldots$ um valor observado ${ }^{z_{t}}$ é usado no instante de tempo $t$. A proximidade destes valores é verificada e a sua probabilidade $\pi\left(z_{k} \mid x_{k}\right)$ é calculada, obtendose assim a importância de cada partícula, através da normalização dos pesos, $\hat{w}_{k}^{i}$. As partículas de maior peso representam as regiões de maior importância da distribuição. Após este passo, 
o processo de seleção é feito, onde é realizada a reamostragem da distribuição anterior $\pi\left(x_{k} \mid x_{k-1}\right)$ das $N$ partículas, usando os pesos $\hat{w}_{k}^{i}$. As novas partículas no instante de tempo $t$, que representam a distribuição posterior, são então obtidas, e chamadas $x_{k}^{i}$. No fim, há o passo de evolução em que, a partir da equação de transição $\pi\left(x \mid x_{k-1}\right)$, novas partículas são geradas de modo a representar a distribuição posterior no tempo $t$.

\subsection{Filtro ASIR}

O filtro de partícula SIR apresenta uma etapa de reamostragem para tentar evitar o problema de degeneração das partículas. Porém, esse processo pode acarretar no empobrecimento da amostra. A utilização de um número finito de partículas é insuficiente para tentar prever o comportamento da cauda da densidade de probabilidade. Esse problema é ainda pior quando não há valores extremos. Para resolver este problema, Pitt e Shephard (1999) introduziram o chamado Filtro de Partículas Auxiliar (APF), na qual este método tem como objetivo aumentar o número de "boas partículas" $\left\{x^{i}\right\}$, considerando que as probabilidades $\pi\left(z_{k} \mid x_{0: k}^{i}\right)$ assumem valores mais altos para essas "boas partículas". A ideia é gerar um conjunto de $N$ partículas aleatórias auxiliares $u_{k}^{\beta^{j}}$, a partir da distribuição inicial $\pi\left(x_{0}\right)$, e calcular a probabilidade de cada partícula auxiliar dado $\pi\left(z_{k} \mid u_{k}^{\beta^{j}}\right)$. Depois disso, $N$ outras partículas são retiradas aleatoriamente a partir da distribuição inicial $\pi\left(x_{0}\right)$. Tais novas partículas são candidatas para representar a distribuição posterior no instante de tempo $t-1$, e nomeadas $x_{t-1}$. Em seguida, a probabilidade $\pi\left(z_{k} \mid x_{k}^{i}\right)$ de cada partícula é calculada. Com estas informações, a importância do peso de cada partícula pode ser calculada e normalizada, por $\hat{w}_{k}^{i}$. O último passo é o passo de evolução, na qual as partículas candidatas para representar a distribuição a pgsteriori no instante de tempo $t$, chamadas ${ }^{x}$, são obtidas, utilizando a equação de transição $\pi\left(x \mid x_{k-1}\right)$.

\section{RESULTADOS E DISCUSÃO}

Nesta sessão são apresentados os resultados obtidos para o modelo de Buckley e Leverett, abrangendo a verificação do problema direto e a estimativa da variável de estado, saturação, com o filtro SIR e ASIR. Os códigos foram implementados para a solução do problema direto e inverso no software Scilab e os resultados gerados em um notebook com processador Intel(R) Core(TM) i5-3337U CPU @ 1.80GHz. No sistema de EDPs acopladas, foi utilizado o método das linhas, com diferenças finitas centrais, e as EDOs foram integradas no tempo com o auxílio da ferramenta ode.

Nesse trabalho foi utilizada uma malha espacial com 101 amostragens espaciais e 51 amostragens temporais no problema direto. Este foi usado como sub-rotina nos filtros para uma amostragem espacial de 51 pontos, para evitar o crime inverso.

Para avaliar o desempenho dos filtros, foi empregado o cálculo do erro quadrático médio (RMS) dado pela Equação 9. 


$$
R M S=\sqrt{\frac{1}{N}} \sum_{i=1}^{N}\left(x_{\text {est }}(t)-x_{\text {teorico }}(t)\right)^{2}
$$

As medidas simuladas foram calculas pela Equação 10, pois neste trabalho, não foram realizados experimentos, sendo usada para acrescentar um ruído gaussiano de $5 \%$ da saturação máxima, obtendo assim as medidas simuladas.

$$
x_{m e d}=x+\sigma \epsilon
$$

onde $x$ é obtido a partir do problema direto com parâmetros conhecidos, $\sigma$ é o desvio padrão das medidas e $\epsilon$ é uma variável aleatória com distribuição normal. A abordagem, que emprega o mesmo modelo matemático para gerar os dados experimentais sintéticos e, posteriormente, resolver o problema inverso, é denominada crime inverso.

Nesse trabalho foram utilizados 50, 200 e 500 partículas para o filtro SIR e 50 para o filtro ASIR como mostra a Tabela 1, junto com os seus resultados para o tempo de CPU e erros RMS. Esses números foram escolhidos de forma aleatória, porque não existe um número adequado, pois cada problema exige certa quantidade de partículas para obter uma boa solução. Estes métodos tem a convergência garantida pelo Teorema dos Grandes Números, ou seja, quando o número de partículas tende ao infinito a probabilidade de convergência é assegurada. No entanto, utiliza-se um número finito de partículas com o intuito de ter uma solução satisfatória e rápida.

Observando a Tabela 1, tem-se que o filtro ASIR com 50 partículas apresenta um tempo computacional de 125,59 segundos e com um erro RMS de 0,0982. Já para o filtro SIR com 500 partículas, o tempo computacional foi de 628,86 segundos e com um erro RMS de 0,1101. Pode-se notar que os erros RMS para estimar a saturação para todos os casos testados foram próximos. Porém, o filtro ASIR apresenta o menor valor comparado com o filtro SIR, para os casos examinados. Por isso, o filtro ASIR apresenta um melhor custo beneficio para a estimação da saturação, já que necessita de um número menor de partículas para ter um bom resultado.

Tabela 1 - Resultado dos filtros SIR e ASIR.

\begin{tabular}{cccc}
\hline Filtros & $\begin{array}{c}\text { Número de } \\
\text { partículas }\end{array}$ & Erro RMS & Tempo (s) \\
\hline \multirow{3}{*}{ SIR } & 50 & 0,1218 & 78,87 \\
& 100 & 0,1112 & 215,96 \\
ASIR & 500 & 0,1101 & 628,86 \\
\hline
\end{tabular}

A Figura 1 apresenta a estimativa da saturação de um reservatório de petróleo, utilizando os filtros de partículas SIR e ASIR, que foram analisadas ao nível de $99 \%$ de confiança, segundo o trabalho de Silva (2010) e Vianna (2009). Nota-se que os dois filtros apresentaram bons resultados para a estimação da saturação, ou seja, os dois métodos 


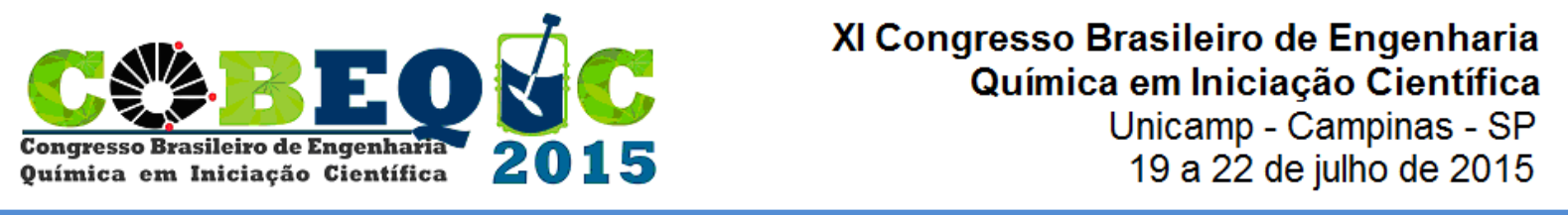

conseguiram filtrar a incerteza das medidas e do modelo, já que para a estimação foi utilizada uma malha diferente. Contudo, o filtro ASIR consegue capturar todos os pontos de saturação dentro do seu intervalo de confiança, fato que não foi possível usando o filtro SIR.

Figura 1 - Estimativa da Saturação usando o filtro (a) SIR com 500 partículas e (b) ASIR com 50 partículas.
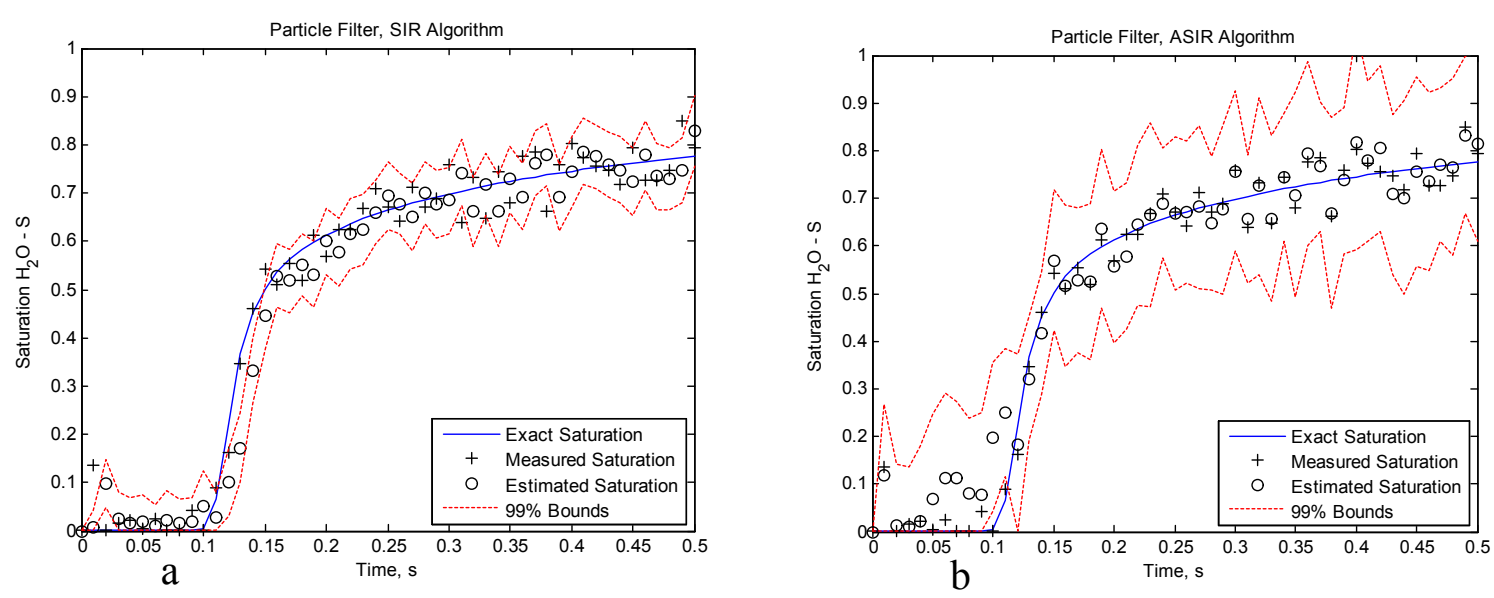

\section{CONCLUSÃO}

Neste trabalho, foram aplicados dois filtros Bayesianos diferentes para um problema de reservatório de petróleo com escoamento bifásico, com o intuito de estimar a saturação com apenas um único dado. Os filtros utilizados foram o SIR (Amostragem por Importância e Reamostragem Sequencial) e ASIR (Auxiliar de Partícula ou Amostragem e Reamostagem por Importância Auxiliar). Os resultados para os dois filtros mostram-se satisfatórios ao estimar a saturação do reservatório de petróleo, entretanto o filtro ASIR apresentou melhores resultados quando comparado com o filtro SIR.

\section{REFERÊNCIAS}

BUCKLEY, S. E.; LEVERETT, M. C. Mechanism of fluid displacement in sands. Petrol. Trans. AIME, vol. 146, p. 107-116, 1942.

KAIPIO, J.; SOMERSALO, E. Statistical and computational inverse problems. New York: Springer, 2004. 
STONE, H. L. Probability model for estimating three-phase relative permeability. Trans. SPE of AIME, v. 249, p. 214-218, 1970.

VIANNA, F. L. V.; ORLANDE, H. R. B.; DULIKRAVICH, G. S. Prediction of the temperature field in pipelines with bayesian filters and non-intrusive measurements. In: 20th International Congress of Mechanical Engineering, Gramado, 2009.

SILVA, W. B.; ORLANDE, H. R. B.; COLAÇO, M. J. Evaluation of bayesian filters applied to heat conduction problems. In: 2nd International Conference on Engineering Optimization, Lisbon, 2010.

PITT, M. and SHEPHARD, N. Filtering via simulation: Auxiliary particle filters, J. Amer. Statist. Assoc., v. 49, n. 446, p. 590-599, 1999. 